\title{
Pasado y futuro del estudio sobre la transición de los jóvenes
}

\author{
Joaquim Casal \\ Rafael Merino \\ Maribel García \\ Universitat Autònoma de Barcelona. Departament de Sociologia \\ joaquim.casal@uab.cat; rafael.merino@uab.cat; maribel.garcia@uab.cat
}

Recibido: 01-07-2008

Aceptado: 15-06-2010

\section{Resumen}

El artículo muestra el estado de la cuestión acerca de los enfoques teóricos en sociología de la juventud. Los autores presentan la sociología de la juventud bajo tres paradigmas o enfoques (el ciclo vital, la generación y la biografía) y toman opción por el tercer enfoque. El primero es muy parejo al desarrollo del funcionalismo, el segundo es muy parejo a los conflictos generacionales a partir de los años sesenta y el tercero es más afín a la sociedad actual y está basado en un enfoque que refuerza una perspectiva próxima al individualismo metodológico y a la segmentación social y cultural de los jóvenes. Los autores mantienen que la perspectiva de la transición profesional y familiar de los jóvenes es un punto de referencia para la sociología de la juventud.

Palabras clave: juventud; transición; biografía; trayectoria; itinerario; inserción; emancipación.

\section{Abstract. Past and Future of the Research on the Transition of Young People}

This paper presents the state of the art concerning theoretical approaches in the sociology of youth. In this paper, the authors outline three paradigms or approaches in the sociology of youth - the lifecycle approach, the generational approach, and the biographical approach - and argue in favour of the third approach. The first approach is closely related to the development of functionalism. The second approach is related to the generational conflicts emerging from the 1960 s onwards. Finally, the third approach best approximates current society, and adopts a perspective that reinforces methodological individualism and the social and cultural segmentation of youth. The authors maintain that the professional and family transition of young adults is a key point of reference in the sociology of youth.

Key words: youth; transition; biography; path; itinerary; insertion; emancipation. 


\section{Sumario}

1. Tres enfoques en la sociología de la juventud

2. La juventud como transición
3. Transiciones, itinerarios y trayectorias

4. Resumen y perspectivas

Referencias bibliográficas

Este escrito pretende resumir en unas pocas páginas algunos de los rasgos que ha caracterizado el enfoque sobre itinerarios y transiciones de los jóvenes en nuestro equipo de investigación social, Grupo de Investigación en Educación y Trabajo (GRET), de la Universidad Autónoma de Barcelona (UAB), desde su fundación, hace veinte años ${ }^{1}$. No se trata de resumir el conjunto de aportaciones teóricas y metodológicas en un balance de las investigaciones realizadas, ni de llevar a cabo una revisión de proposiciones formuladas por los autores en años anteriores, ni de fundamentar la aportación del enfoque que llamamos «transición» a los estudios sobre sociología de la juventud. El objeto de estas páginas consiste en bosquejar el futuro de este tipo de análisis en relación con una mayor comprensión de tendencias sociales emergentes sobre juventud, tanto en países o zonas centrales del capitalismo informacional, como en zonas periféricas y de exclusión, resultante de los procesos de globalización en lo económico y lo cultural. No es propiamente un balance de investigaciones rea-

1. El GRET (www.uab.cat/gret; tel.: 0034 935811789) nació en el año 1987 como grupo estable de investigación en la Universidad Autónoma de Barcelona, en el marco institucional del Instituto de Ciencias de la Educación. Inicialmente, tomó como nombre de referencia el de GRT (Grupo de Investigación sobre Transición) para adoptar finalmente el nombre de GRET por su vinculación al estudio de la relación específica entre educación y trabajo. Posteriormente, ubicó sus dependencias en la Facultad de Ciencia Política y de Sociología. Este equipo de investigación ha definido desde el principio tres campos de análisis social: la relación entre educación y trabajo en el marco de los estudios comparados, el papel de la universidad en la formación superior y la inserción social y profesional de los jóvenes. En los años ochenta, destacan tres grandes encuestas longitudinales a jóvenes de 19, 25 y 31 años que fructifican poco después en dos tesis doctorales acerca de la construcción de la formación y la emancipación familiar y dos publicaciones (La inserción social y profesional de los jóvenes, CIDE-MEC, 1991, y Los hombres y las mujeres de 31 años de edad, ICE-UAB, 1989). El artículo más citado acerca de las modalidades de transición es del año 1996 y se publicó en el número 75 de la revista REIS («Modos emergentes de transición a la vida adulta en el umbral del siglo XXI: aproximación sucesiva, precariedad y desestructuración»). En el cambio de siglo, destaca la encuesta a los jóvenes en Cataluña, dos investigaciones acerca de la transición de la escuela al trabajo en España (Enquesta als joves de Catalunya 2002: Itineraris educatius, laborals i familiars, Generalitat de Catalunya, 2004; Transición escuela-trabajo contratos $i+d+i$ digicyt; transición 16-19 años y análisis socioestadístico de la encuesta ine-etefil 05) y los artículos publicados en el número 79 de Papers: Revista de Sociologia, del año 2006 («Aportaciones teóricas y metodológicas a la sociología de la juventud desde la perspectiva de la transición» y «Changes in forms of transition in contexts of informational capitalism»). El presente artículo obedece, entonces, a la reflexión y a las propuestas que se emiten desde la perspectiva de la transición de los jóvenes, sobre todo en contextos del capitalismo informacional, y tiene su marco de referencia en estos escritos anteriores. 
lizadas, pero supone una clarificación de enfoques o direcciones; en definitiva, el artículo pretende indicar el desarrollo futuro del enfoque de la transición.

El artículo consta de tres apartados. En el primero, se constata la debilidad y la caducidad de algunos referentes teóricos (desde la perspectiva estructuralfuncionalista, hasta determinadas proposiciones en el marco de la postmodernidad) en el análisis de la situación social de los jóvenes, dada la expansión del capitalismo informacional, sobre todo en zonas ricas o centrales, y se propone realitzar el enfoque sobre itinerarios y transiciones como perspectiva basada en la crítica a la linealidad de las transiciones de antes (las propias de la industrialización), pero también crítica ante el auge de perspectivas basadas en la reversibilidad ante las transiciones lineales del capitalismo anterior ${ }^{2}$, ya que se centra en la complejidad de las transiciones y los efectos del capitalismo informacional sobre ellas. El artículo considera, en este apartado, la existencia de tres enfoques distintos acerca de la sociología de la juventud (como etapa de la vida, como generación y como transición), proponiendo este tercer enfoque como el más certero para un marco teórico de la sociología de la juventud. En segundo lugar — en un segundo epígrafe- - se hace referencia a la juventud como transición, tanto en lo laboral como en la emancipación familiar como elementos específicos y centrales, distinguiendo entre la condición social de la juventud (la transición en sí misma, como concepto) y la situación social de los jóvenes en relación con las modalidades de transición (cambios en la transición, sobre todo en relación con el auge del capitalismo informacional). Finalmente, en el último apartado, se hace referencia más detallada a los términos utilizados en el campo de la investigación social en el enfoque de los itinerarios y, en cierta forma, expresa no sólo una línea de investigación, sino también un plan de trabajo futuro acerca del análisis sociológico de la juventud desde el GRET. Es por esto que, en sus páginas finales, se propone el análisis longitudinal en el estudio de las transiciones de los jóvenes y sus impactos en la socialización de ellos ${ }^{3}$.

\section{Tres enfoques en la sociología de la juventud}

El GRET se constituye como grupo estable de investigación sobre la transición de los jóvenes a la vida adulta en el año 1987 y percibe prontamente la obsolescencia de la mayor parte de los supuestos y de las perspectivas teóricas y metodológicas de la sociología de la juventud, sobre todo en rela-

2. Ciertos pensamientos que podrían inspirarse en formas de representación de la postmodernidad (todo es líquido, por ejemplo, en el enfoque de Z. Bauman) pueden chocar con la sociología basada en el análisis de la transición de los jóvenes. Ciertamente, las transiciones no son lineales en el capitalismo informacional, pero tienen marca social y están sujetas a ciertas irreversibilidades en términos de efectos sociales. Se trata de una posición defendida desde el GRET como crítica a las "transiciones yo-yo", donde se enfatiza en los aspectos de reversibilidad ante la incertidumbre y las reorientaciones (ver, por ejemplo: J. Machado Pais, Chollos, chapuzas, changas, Barcelona, Anthropos, 2007).

3. En cierta forma, la perspectiva de la transición toma aspectos de la teoría de roles y del ciclo vital y aspectos de la propuesta del análisis generacionalista. En uno de los diagramas de Lexis (una adaptación), es un referente metodológico de mucho valor comparativo. 
ción con el contexto de los años ochenta (es decir, como un desfase entre los marcos teórico y contextual). La constatación inicial del GRET queda reforzada al considerar que, en los años ochenta, hay cambios importantes por parte de los jóvenes en la forma de construir el futuro (tanto profesional como familiar). Esta realidad se había percibido ya bastante tiempo antes y quedó reflejada en esta revista de sociología, puesto que se editó en ella un número monográfico sobre juventud hace más de veinte años ${ }^{4}$. La proposición que sostenemos es que, en sociología de la juventud, las aportaciones de los sociólogos pueden resumirse, de forma explícita o implícita, en tres enfoques (o paradigmas): el ciclo vital o la juventud como etapa de la vida; las generaciones en conflicto o la juventud como radicalismo, y, finalmente, la juventud como tramo biográfico de jóvenes en transición. Se trata de una consideración de paradigmas que no es clasificatoria ${ }^{5}$ y sólo pretende diferenciar el enfoque biográfico de itinerarios y transiciones como tal y subrayar su potencialidad interpretativa en el marco del capitalismo informacional. Es decir, que, en el actual contexto de cambios económicos sociales y culturales inducidos del capitalismo informacional, el enfoque de la transición se revela como muy interesante en la propuesta teórica y metodológica, sobre todo por su enfoque como proceso social (terreno muy propio del constructivismo social), por los contreñimientos sociales (terreno muy propio del análisis acerca de las desigualdades) y por su anclaje en lo longitudinal (terreno muy propio de los itinerarios y las trayectorias), es decir, por tratarse de una propuesta basada en el individualismo metodológico ${ }^{6}$.

El contexto social de los jóvenes en los años ochenta resulta muy diferente de los contextos anteriores, sobre todo es generador de nuevas contradicciones. Principalmente, por la segunda escuela de masas ${ }^{7}$ promotora de expectativas de movilidad social y receptora de demandas sociales de educación escolar

4. Papers: Revista de Sociologia, 25 (1985), Universidad Autónoma de Barcelona. La primera parte consiste en una relectura del ensayo de Aranguren acerca de la juventud europea con una participación directa de dicho autor; la segunda parte reflexiona acerca de la juventud como objeto de la sociología, con contribuciones de Grootings, Casal y Cardús; la tercera parte aborda la transición de la escuela al trabajo con aportaciones de Planas, Welbers y Francchi.

5. Cada sociólogo ha tenido, a lo largo de su vida profesional, tentación implícita o explícita de categorizar la teoría sociológica según enfoques o paradigmas. La clasificación de Ritzer, seguramente la más citada y conocida, tiene por misión identificar y diferenciar escuelas y autores. En este artículo, los autores no pretenden hacer una clasificación, sino, simplemente, ilustrar como el enfoque biográfico, de los itinerarios y las transiciones sobre el que venimos trabajando, tiene potencialidad para explicar la situación de los jóvenes en el marco del capitalismo informacional.

6. Ver, entre otros, J. Noguera (2003), «¿Quién teme al individualismo metodológico?», Papers: Revista de Sociologia, 69.

7. En el GRET, entendemos como la "primera escuela de masas» la generalización de la escolarización propia de la postguerra mundial (en España, con retraso). Para nosotros, la segunda escuela de masas hace referencia propiamente a la prolongación de los estudios superiores fruto de la comprensividad y la demanda satisfecha de educación escolar propias de los años sesenta y setenta (para España, los ochenta, iniciada años antes con los pactos de la Moncloa y culminada en los gobiernos socialistas de los años ochenta y noventa). 
prolongada, junto con la crisis económica que repercute en un mercado de trabajo basado en una contracción laboral y el aumento del paro juvenil, o los dos aspectos juntamente. Todo ello relacionado con la presión demográfica acerca de las cohortes de edad de las promociones de jóvenes y los efectos sociales vinculados a formas o estilos de vida con cierta desafección social. De ahí, entonces, que emerjan como temas objeto de interés periodístico la nueva delincuencia juvenil, las tribus urbanas, la abstención electoral, las toxicomanías, la emergencia de lo musical o los estilos de vida diferenciados o, en fin, la tensión entre grupos de iguales o entre los jóvenes y los llamados «adultos». Una serie de variaciones en conductas y percepciones que emergen en un contexto de cambio y permanencia ${ }^{8}$.

Así ha sido como la emergencia social de lo juvenil como algo diferenciado ha sido objeto de interrogación por parte de los medios de comunicación y de los científicos sociales: ante la emergencia de grupos neofascistas, los medios se han interrogado acerca del racismo latente de algunas tribus urbanas excluyentes o acerca de jóvenes organizados en bandas rivales; el abstencionismo político ante los procesos electorales ha sido otro referente del análisis acerca de la desafección de los jóvenes o acerca del alejamiento de éstos de las instituciones religiosas y sus tendencias hacia la secularización o sus conductas morales al margen de las pautas morales de la jerarquía religiosa; la morbilidad de los jóvenes ha sido también campo de atracción ante la emergencia del consumo de estupefacientes o acerca del riesgo en los estilos de vida. De alguna forma, entonces, la juventud se ha hecho merecedora de un lugar en la actividad periodística y del ensayo, aunque con nuevos temas o dianas (antes del 68, por ejemplo, ha sido objeto del periodismo la cooptación de los jóvenes en encuadramientos políticos o sindicales, militares o paramilitares, religiosos o laicistas, en instituciones formales o informales, etcétera; poco después, la contracultura se ha hecho eco en temas culturales y políticos; poco después, un cambio temático entorno al paro juvenil y la delincuencia y la transgresión de menores, y, después, nuevos temas acerca del retraso en la emancipación familiar o la autonomía cultural y de las formas de vida de los jóvenes). La particular percepción positiva o negativa de acciones atribuidas preferentemente a los jóvenes por parte de los "adultos» ${ }^{9}$ ha generado cuestiones de claro enfoque adultocrático, mientras que los jóvenes se han apegado a un discurso donde se subraya la especificidad de lo juvenil, como si se abonara de nuevo el conflicto entre generaciones.

Por otra parte, el Estado del bienestar ha generado mientras tanto una verdadera batería de acciones conducentes a las llamadas «políticas de juventud»,

8. J. Casal, J.L.Aranguren, J. Carandell, O. Homs y A.Puig (1985), «La joventut a Europa 25 anys després: Una relectura d'un vell assaig», Papers: Revista de Sociologia, 25.

9. El término adulto ha sido objeto de crítica por parte del GRET, por sus raíces en formas de psicologismo propios de la psicología de la educación basados en A. Gessel (los estadios en el desarrollo del niño). El término adulto simplemente lo utilizamos como sinónimo de «no-joven», en el sobreentendido de que estos términos pueden dar lugar a debate teórico o, en todo caso, a malentendidos. 
primeramente basadas en un acercamiento institucional, la participación y los servicios específicos y, posteriormente, en políticas de apoyo al sistema educativo, al fomento de la formación para el empleo y el salario social en muy variadas formas ${ }^{10}$. La emergencia de lo juvenil (básicamente, la escuela de masas y formas autónomas de percepción de lo cultural y social) ha supuesto, entonces, que las ciencias sociales vean a la juventud como objeto de estudio.

Así, las disciplinas sociales han tenido en cuenta, en sus investigaciones y lugares propios ${ }^{11}$, una referencia al término juventud. Se han visto impulsadas a considerar como fenómeno social a tener en cuenta la emergencia de la adolescencia en los entornos sociales industriales. Por ejemplo: la historia haciendo una referencia a la aparición de los jóvenes como actores diferenciados y protagonistas; la antropología cultural haciéndose eco del debate entorno a los ritos de paso a la sociedad adulta entre poblados indígenas; la pedagogía acerca de la emergencia del adolescente y del joven en una escuela en extensión; la ética acerca de las formas morales de conducta de los jóvenes una vez escindidos del caparazón del autoritarismo parental o intelectual; la psicología evolutiva acerca de la construcción de la identidad del yo y la crisis de la adolescencia, o las disciplinas vinculadas a la expresión (deportiva, musical, artística, representativa, etc.) acerca de la creatividad específica de los jóvenes. Es decir, que, en cada disciplina, el término juventud ha logrado cierta presencia ${ }^{12}$.

Es por todo ello que el arranque del GRET en la investigación social sobre jóvenes tiene en su origen dos objetivos: primeramente, dotar a la sociología de la juventud de un enfoque teórico más acorde con los cambios de fin de siglo y, en segundo lugar, experimentar nuevos procedimientos metodológicos menos centrados en la percepción de valores y actitudes de los jóvenes y más focalizados en la construcción de itinerarios y realización de las transiciones.

10. Durante la larga recesión económica de los años setenta, ha habido una efectiva retención de futuros activos en el sistema de enseñanza escolar, una proliferación de ayudas a dispositivos de inserción profesional (formativos, informativos), un fomento del cooperativismo y la autoocupación, ayudas directas mediante salarios sociales $\mathrm{u}$ otras ayudas y plataformas para la reinserción social y sanitaria, es decir, un conjunto de ayudas directas o indirectas por parte del Estado (llamadas "políticas de transición»), basadas casi exclusivamente en aspectos sociolaborales; posteriormente (en la actualidad), las políticas de transición desarrollan también acciones sobre la vivienda de los jóvenes prestos a emanciparse).

11. Por «lugares propios» entendemos los espacios de actividad generados en el marco de una disciplina: manuales, ensayos, planes de estudios, formaciones específicas, columnas o editoriales, escritos, redes internacionales, proyectos, etc. En las disciplinas, no sólo hay cabida para las investigaciones, sino también para este conjunto de actividades, sean docentes, de investigación o de difusión. Algunas disciplinas (por ejemplo: la economía y el derecho) y algunos manuales no hacen referencia directa a la juventud o bien tratan el tema de forma tangencial. También ocurre esto en la misma sociología.

12. Personas apegadas a lo juvenil, tanto desde la intervención política y social como al estudio y la formación, reciben el nombre de «juvenólogos». Se trata de un lugar común y muy interdisciplinario, donde la consulta bibliográfica resulta un instrumento de interés al ver referenciados continuadamente autores primeramente como Aries, Erikson o Mead. Posteriormente, como Rappoport, Allerbeck o Laslet. Y, últimamente, como Feixa, Reguillo o Galland. 
La biografía, la perspectiva longitudinal y la centralidad de las transiciones han sido los vectores teóricos y metodológicos de tantos años de investigación y de contraste con los actores y los agentes ${ }^{13}$. Es más, a nuestro juicio, estamos en la confianza de que tales vectores pueden reportar, en años sucesivos, elementos de comprensión de la juventud y, por tanto, estamos en la confianza de que han de ser considerados centrales en la investigación sociológica sobre juventud ${ }^{14}$.

La sociología de los últimos treinta años se ha nutrido casi exclusivamente de las perspectivas del ciclo vital y el conflicto entre generaciones. El enfoque biográfico y de los itinerarios que proponemos es relativamente reciente y busca formas de superación de la secular polarización teórica entre integracionalistas y conflictivistas, incorporando algunos elementos que pueden contribuir a comprender el fenómeno actual de las transiciones sobre trabajo y emancipación. La perspectiva de los itinerarios y las biografías busca definir un campo de conceptos y relaciones que hagan posible interpretar y comprender mejor los cambios y sus repercusiones sobre los jóvenes y su juventud. Esta es la perspectiva del GRET. Puede ser que, en pleno industrialismo, el planteamiento funcionalista fuera atractivo y de interés para la sociología ${ }^{15}$; para la sociedad convulsa del crecimiento acelerado de los sesenta y la crisis ideológica de los setenta, parece que el planteamiento generacionalista también podría resultar atractivo e interesante, sobre todo por el acierto de contraponer lo «viejo» y lo «nuevo». Finalmente, para la sociedad del informacionalismo (caracterizada por la turbulencia y el cambio), parece que el planteamiento biográfico también puede resultar particularmente pertinente y de interés, sobre todo atendiendo al hecho del constructivismo social y las rupturas biográficas en lo público y en lo privado ${ }^{16}$. En fin, tres perspectivas de análisis distintas, tres paradigmas,

13. Ciertamente, la investigación social comporta largos tiempos de soledad y elaboración de los investigadores (tiempos de estudio, de planificación y búsqueda, de partenariado y elaboración, de supervisión o validación, de análisis de datos y de redacción de informes, de difusión científica, de contraste). Pero el estudio de las transiciones de los jóvenes nos ha permitido realizar contactos con actores y agentes sociales (profesionales de la intervención social, promotores y gestores de las políticas sobre jóvenes, etc.) y con jóvenes constructores de transiciones. Todo ello ha repercutido positivamente en beneficio nuestro, ya que han tenido mucho protagonismo en la confección de nuestras ideas o propuestas.

14. Muy probablemente, cada disciplina podría hacer un ejercicio similar. Así, la psicología evolutiva y de la adolescencia podría presentar tres enfoques: el freudismo, el interaccionismo y el gessalismo.

15. Es interesante recordar como algunos autores incidieron fuertemente en la contraposición entre sociología burguesa y marxismo. El mismo I. Zeitlin planteaba la sociología disciplinaria como un ejercicio prolongado de réplica al discurso de Marx (Ideología y teoría sociológica, Amorrortu). Posteriormente, otros autores han reafirmado esta línea de pensamiento.

16. Buena parte de los ensayos acerca del cambio social derivados del hecho de la globalización se hacen eco de su significación en el campo de lo biográfico. Muchos autores se centran en un análisis acerca de la estructura económica y política mundial (como S. Amin en El capitalismo en la era de la globalización o Chomsky y Dieterich en La aldea global), pero otros abren espacios de reflexión acerca del cambio de valores. El ensayo de Gil Calvo 
tres enfoques muy complementarios, aunque muchas veces en franca contraposición.

\subsection{Primer enfoque: la juventud como ciclo vital o etapa de la vida}

La figuración de la juventud como un ciclo vital está muy emparentada con los primeros pasos de la sociología y sus puntos de contacto con la psicología evolutiva. Básicamente, la sociología del ciclo vital propone la existencia de cuatro grandes etapas (infancia, juventud, vida adulta y vejez) y, dentro de cada ciclo, algunas subdivisiones para atender a la diversidad de situaciones (la primera y la segunda infancias, la adolescencia y los jóvenes adultos, el estadio de matrimonio, la crianza y los roles parentales, los nidos vacíos, la jubilación y la decrepitud o cuarta edad). Las hipótesis de los estadios de la persona (con vínculos en la psicología evolutiva) y la del ciclo de reproducción familiar (arraigadas en la sociología de la familia) han sido los principales referentes ${ }^{17}$ de este enfoque.

La perspectiva de los ciclos vitales ha sido una aportación fecunda y repetida; adolescencia y juventud como una etapa de la vida —más o menos larga, más o menos diferenciada, más o menos conflictiva- basada en criterios de superación de ciclo: abandonar determinadas pautas más propias del ciclo anterior (la infancia, en este caso) en favor de la adquisición de pautas más propias de la vida adulta (trabajo, familia propia y reconocimiento social). Muchas veces, además, los cambios de ciclo irían acompañados de certificación social o ritos de paso, aspectos que han llamado mucho la atención de la antropología cultural ${ }^{18}$. Los manuales de sociología general, cuando hacen referencia a los adolescentes, tienden a centrarse en la perspectiva del ciclo

(El destino) o de U. Beck (Hijos de la libertad), entre otros, resultan emblemáticos. Otros autores se centran más exclusivamente en una reflexión del cambio de la postmodernidad sobre las conductas y las actitudes de los jóvenes (A. López-Blasco, con otros autores como J.A. Machado, M. de Bois o Walther y Stauber o.c.), sobre todo acerca de las «transiciones yo-yo» a las que nos hemos referido anteriormente. El escrito de Sennet acerca de la corrosión del carácter también es una obra referente.

17. La psicología evolutiva ha tenido como misión fragmentar la primera y la segunda infancias en muchas fases y describir los atributos y los desarrollos previstos para cada uno de ellos (p.e. Gessel). Parte de este enfoque fue transportado a la psicología de la adolescencia y de la juventud con el fin de fragmentar y describir mejor los procesos de construcción de la identidad personal. Primeramente, Hall y después Erikson (en una perspectiva psicoanalítica) y muchos otros autores trabajaron en esta dirección. Si infancia y adolescencia resultaban terreno atractivo para la psicología evolutiva, el análisis de los roles parentales en la familia y también de la vejez lo fue para la sociología. Así se fue cumpliendo una prefiguración de todos los ciclos vitales, aunque sin poder sobrepasar el carácter eminentemente descriptivo y etnocéntrico. Piaget ha retomado de forma más epistemológica aspectos de evolución y construcción de la personalidad.

18. M. Mead ha sido considerada como un referente en el estudio de la adolescencia en zonas tribales. Cultura y compromiso (Buenos Aires, Granica, 1970) se ha convertido en un libro clásico. Conviene también, en este tema, leer lo que J.L. Zárraga escribe en el Informe Juventud en España 1984 (Madrid, Ministerio de Cultura, 10 a 16). 
vital y la distancia entre la atribución de roles de adulto y la asunción de la capacidad reproductiva ${ }^{19}$.

En la perspectiva del ciclo vital, la sociología ha tenido un interés muy explícito en interpretar las especificidades del rol de joven y la relación entre padres e hijos, en un contexto social de modernidad, de industrialismo y urbanismo. El análisis sociológico, así, versa sobre las distancias entre los logros psicofísicos en la pubertad y el retraso en asumir responsabilidades sociales plenas. Es decir, plena fuerza física y de impulso sexual versus la distancia de reconocimiento social de adultez a causa de la formación escolar general y prolongada, la prioridad de la ocupación laboral estable, el matrimonio como forma de vida en pareja o la preparación del hogar. Según esta manera de representar el hecho juvenil, en muy poco tiempo, los púberes conseguirían estatuto de adulto en términos físicos (madurez sexual y plena atribución de fuerza física, principalmente), pero serían expropiados del acceso al mundo de los adultos hasta al cabo de unos años (profesión definida, vivienda propia, matrimonio de elección, nupcialidad formalizada, etcétera). El desajuste de logros y de responsabilidades en el tiempo sería la razón de fondo de los descontentos y las tensiones de los jóvenes, y la relación entre adultos y jóvenes sería de malestar, de crisis paterno-filial o crisis generacional propiamente dicha ${ }^{20}$.

Generalmente, la percepción se ha vestido de referentes adultocráticos ${ }^{21}$. En esta perspectiva, la juventud sería pensada como un tiempo vacío o de espera solo evaluable positivamente en función de la asunción de roles propiamente de adulto (llegar a una profesión, oficio o trabajo de continuidad, conseguir una pareja estable, ahorrar para acceder a una vivienda propia, asistir a las celebra-

19. Por ejemplo: A. Giddens (1989), Sociología, Alianza, 116, o bien Macions y Plummer (1999), Sociología, Prentice-Hall, 145 i 146. En general, la sociología de la juventud, cuando aparece en los manuales de sociología, lo hace en estos términos. En la literatura más especializada, se reemprende este discurso (por ejemplo: Allerberck y Rosenmayr). Más adelante se acuña la expresión «nueva etapa de la vida» en la propuesta teórica de $\mathrm{O}$. Galland.

20. Algunos libros clave en esta dirección son: L. Rappaport, La personalidad desde los 13 a los 25 años, Buenos Aires, Paidós, 1986 (traducción del original de 1972); E. Erikson, Identidad, juventud y crisis, Madrid, Taurus, 1989 (traducción del original de 1968), y Sociedad y adolescencia, Madrid, Siglo XXI (traducción del original de 1960). Este enfoque ha sido muy bién explicado por Allerberck y Rosenmayr (Introducción a la sociología de la juventud, Buenos Aires, Kapesluz, 1979). Los manuales de sociología también han acompañado este enfoque durante muchos años (por ejemplo: Horton y Hunt, Sociología, México, McGrawHill, 1986). Laslett hizo famosa la expresión «el narcicismo radical de los jóvenes», no sólo para describir su ensimismamiento, sino también para expresar como el radicalismo es pasajero ante la integración en la estratificación social y las pautas culturales dominantes.

21. Conviene tener en cuenta que las aportaciones de Durkheim, como sociólogo principal en la primera sociología de la educación y la juventud, tenía un componente adultocrático muy considerable. La sociología más vieja y ya tradicional ha tendido a conceptuar la «socialización» como la acción de interiorización de la realidad de los adultos a partir de la acción educativa de los mismos adultos. En general, todo el estructuralismo funcionalista, tan importante en la sociología, se ha fundamentado en esta perspectiva. 
ciones o fiestas familiares, etcétera). Contrariamente, la ausencia de profesión u oficio (estudios largos), la sexualidad no estable, el gasto económico inmediato o bien la insumisión paterno-filial serían vistas como evaluaciones negativas de las formas de ser joven. Dentro de este planteamiento (enfoque adultocrático), los ritos y los símbolos de paso mencionados anteriormente no serían más que señales y mensajes que los adultos emitirían recurrentemente a los menores a favor del crecimiento y la adultez ${ }^{22}$.

Por todo esto, en la perspectiva del ciclo vital, se ve un marcado carácter negativo en favor de los roles y el estatus de adultez: los jóvenes son pensados desde la indeterminación y la moratoria en la toma de roles ${ }^{23}$. La negatividad, expresada así, puede tener dos concreciones, bien como acusación, bien como exculpación. En ambos casos, sin embargo, se acaba considerando que el mundo de los jóvenes es extraño al mundo de los adultos: poco comprensible, poco entendedor, poco justificable, desconocido, sin «oficio ni beneficio», sin techo, sin raíces, sin estabilidades, y se respalda la idea de que los jóvenes forman generaciones en tensión con los adultos. Es por eso que, en paralelo a este enfoque, emergió, ya hace muchos años, la perspectiva de las generaciones.

\subsection{Segundo enfoque: la juventud como generación en conflicto}

La segunda perspectiva sobre juventud se focaliza en el hecho "generacional», sobre todo desde el conflicto entre generaciones de jóvenes y adultos. Inicialmente, proviene de Mannheim, pero tiene su máximo esplendor en el análisis de la contracultura durante los años sesenta. La perspectiva de las generaciones en tensión fue construida hace bastantes años ${ }^{24}$. Según ésta, los jóvenes representarían los valores asimilados al cambio social y el progreso en detrimento de los adultos y ancianos, que representarían los valores asimilados a la tradición y a la identidad étnica y geográfica. Paralelamente, y en tensión con este sector demográfico, los jóvenes representarían también las tendencias a la anomia, mientras que los viejos representarían los valores más inmutables y seguros ${ }^{25}$. A menudo, también desde un enfoque más rupturista, se ha afianzado la perspectiva de las generaciones, sobre todo al elevar a la juventud a la categoría de "nueva clase social ascendente y revolucionaria», como ocurrió en ciertos análisis de la realidad juvenil en los años sesenta. No solo la perspectiva funcionalista ha estado implicada en la perspectiva gene-

22. Hay que decir que, en la socialización de los adultos sobre los niños, existe una recurrencia en mensajes de adultez para estimular el crecimiento de los menores. Una propensión que hoy en día choca estrepitosamente con las hipótesis sobre el complejo de Peter Pan.

23. 23. O. Galland, en Sociologie de la jeuneusse (París, Colin, 1991), usa el término moratoria, pero nos parece que es muy cercano a la teoría de roles.

24. Los medios de comunicación se han hecho eco de la revuelta de los estudiantes universitarios de mayo del 68 en París.

25. K. Mannheim fue el referente para la sociología de las generaciones que posteriormente se ha divulgado más (M. Jensen, La teoría de las generaciones y el cambio social, Madrid, EspasaCalpe, 1976). 
racionalista, también se ha hecho eco en la hipótesis de la contracultura ${ }^{26}$. La perspectiva de las generaciones se basa en cuatro aspectos: la descripción de las rupturas, la propuesta de la diferenciación de las subculturas juveniles, la hipótesis entorno del narcisismo radical de los jóvenes y la consideración de la «juventud positiva».

La descripción de las rupturas entre generaciones es la vertiente más periodística y atractiva de la descripción «diferencial» de los jóvenes: las generaciones serían los conductores de las rupturas sociales y del cambio; tantas rupturas, tantas generaciones. Las subculturas juveniles es la vertiente más extensa en los estudios sobre cambios y tensiones entre los jóvenes, inicialmente por influjo de la antropología cultural, pero después sociológicamente arraigados a las teorías de la reproducción cultural o el viejo enfoque de la «cultura de masas» y una cultura juvenil de confrontación (alternativas, individualistas o colectivas). El narcisismo radical de los jóvenes sería una tercera perspectiva, muy conocida y formulada inicialmente en el contexto funcionalista, aunque después fue empleada desde posiciones más radicales o alternativas. Es el contrapunto de la teoría del sesgo de responsabilidades y un intento de explicar la emergencia de la contestación y de formas subculturales extrañas al mundo de los adultos: la hipótesis del radicalismo de los jóvenes ${ }^{27}$ (la contracultura, el antiautoritarismo y el comunitarismo).

Pero poco tiempo después, sin embargo, la crisis del capitalismo industrial en favor del informacionalismo, la caída del comunismo y la emergencia del pensamiento utilitarista y del pensamiento único, los desajustes entre formación y trabajo, el individualismo, o la caída de expectativas de éxito, ponen éstas hipótesis en duda; probablemente, el cambio social resulta imparable incluso para las mismas hipótesis. Finalmente, la descripción de la «nueva condición juvenil» ${ }^{28}$ puede considerarse como una reactivación de la idea de narcisismo, ya que presenta la «nueva condición juvenil» como una fase larga

26. Ya hace unos años fueron particularmente leídos Th. Roszak, El manifiesto de una contracultura (Kairós, 1972, traducción del original de 1968) y G. Mendel, El manifiesto de la educación (Madrid, Siglo XXI, traducción del original de 1973).

27. Las aportaciones desde el psicoanálisis siempre han tenido una cierta resonancia desde la sociología. En el caso del «narcisismo», se trata de una importación del conocido mito hacia la sociología de la juventud en el momento en que se producían cambios radicales en los años sesenta. El mismo funcionalismo (con Laslett) incorpora por analogía la idea de narcisismo de los jóvenes, que la eleva a la categoría de radicalismo (el narcisismo radical de los jóvenes). Pocos años después, $\mathrm{H}$. Mendel incorpora el concepto de radicalismo contracultural desde una perspectiva más crítica. También es conocido que otros autores han trabajado sobre la idea (por ejemplo: A. de Miguel) de que el radicalismo es más una cuestión temporal ligada a las edades y muy a menudo más superficial que real. La literatura sobre el tema tuvo un reflujo con motivo de la crisis del mercado de trabajo y, posteriormente, ha resucitado con motivo de la emergencia de los movimientos antiglobalización.

28. La propuesta de la juventud afirmativa que se ha construido en documentos difundidos recientemente (C. Martí, J. Coll, J. Casanoves y otros) es de difícil ubicación en este planteamiento a tres bandas, porque, según como, enraíza con las hipótesis de ciclo vital, pero con modificaciones substanciales. Por eso se considera aquí, sobre todo por el carácter reivindicativo del hecho juvenil, como propuesta afirmativa. 
y prolongada de creatividad y permanencia en la subcultura juvenil como una nueva manera de vivir el individualismo.

Tanto el primer enfoque como éste, parten de una consideración común acerca del carácter sociohistórico de la juventud, con tinte muy esencialista. Es en este marco general de la realidad juvenil como construcción sociohistórica que emerge el tercer enfoque.

\subsection{Tercer enfoque: la juventud como tramo biográfico de transiciones}

El tercer enfoque teórico analiza el hecho biográfico de las personas articulando algunas aportaciones del neomarxismo y el enfoque estructural, el interaccionismo simbólico y el constructivismo social. Desde el punto de vista metodológico, propone un enfoque biográfico y longitudinal que contribuye a conseguir una mejor comprensión de los procesos biográficos de los jóvenes; desde el punto teórico cercano a las tesis de la segmentación sobre el mercado de trabajo y enraizado con las transiciones laboral y familiar, sobre todo en la perspectiva de los itinerarios de transición y las trayectorias sociales. La perspectiva de la transición se ha construido, pues, como tercera vía teórica, a fin de superar algunos de los tropiezos o sesgos heredados de los otros dos enfoques, sin caer en pretensiones ni eclecticismos fáciles, y encontrar un camino que permita un enfoque de la juventud más sociológico, más político y también más próximo a las elecciones racionales y a las emociones de los actores. Cabe decir que hay una cierta congruencia entre esta perspectiva y las formas o modalidades de transición emergentes en el capitalismo informacional ${ }^{29}$.

El punto de partida es el actor social como sujeto histórico y protagonista principal de la propia vida que articula de forma paradójica y compleja la elección racional, las emociones, las constricciones sociales y culturales y las estrategias de futuro. El tema de los jóvenes y la juventud no es solo un conflicto de roles, ni tampoco un conflicto entre generaciones; la perspectiva biográfica procura integrar estos aspectos en la concepción de itinerario y de trayectoria. El enfoque de la teoría de los roles ha surgido de una raíz muy estructural. La perspectiva del conflicto generacional ha tenido raíces en el análisis del cambio social y cultural. El enfoque de las biografías y los itinerarios procura una triangulación a tres bandas: la sociedad como estructura, los hombres y las mujeres como actores y las generaciones como resultantes de procesos históricos de cambio. En definitiva: estructura, acción y historia como proyecto de trabajo sociológico centrado, en este caso, en los jóvenes y la construcción de lo social.

La juventud se entiende, desde este enfoque, como un tramo dentro de la biografía, que va desde la emergencia de la pubertad física hasta la adquisición de la emancipación familiar plena y desde la salida del sistema escolar hasta la

29. Resaltamos el carácter central de este aspecto. Donde formulamos de forma expresa la hipótesis acerca de las modalidades de transición fue en la REIS de 1996. 
inserción laboral (posición y enclasamiento); es decir, transición profesional y familiar (y la desigualdad social en sus logros). Como nuestro contexto histórico pasa por el cambio domiciliar respecto a la familia parental o de origen (dimensión neolocal), la juventud no es otra cosa que un proceso social de autonomía económica y emancipación familiar plena que concluye con el acceso a un domicilio propio e independiente ${ }^{30}$. Es, pues, una concepción de juventud que adopta algunos aspectos de la teoría de roles y que incorpora la tensión familiar entre hijos y padres, pero que se focaliza en el proceso de adquisición, enclasamiento y emancipación familiar plena: un proceso social que tiene lugar en un determinado tramo biográfico (las edades de los jóvenes) ${ }^{31}$.

En esta construcción conceptual, hay términos clave: itinerario, trayectoria y transición (en el aspecto laboral y en la emancipación). El siguiente apartado nos remite a la dimensión biográfica de los individuos jóvenes que realizan la transición y que describen itinerarios y trayectorias (de trabajo o familia), el paso de la escuela al trabajo y el proceso de emancipación familiar. En el artículo en castellano de la revista Papers de Sociología número 79 incidíamos más sobre el lugar de los itinerarios; en este escrito nos centramos más en la transición como tal.

\section{La juventud como transición}

La crisis del mercado del empleo de los años setenta y ochenta abonó la emergencia de muchos diagnósticos sobre los jóvenes centrados en las dificultades de acceder a empleos esperados. Los informes sobre transición al trabajo abundaron principalmente en el contexto europeo, donde los estados y la UE

30. Cabe tener en cuenta escritos nuestros anteriores en relación con la natalidad primera. Puestos a establecer límites, la primera natalidad (o adopción o similar en su caso) es un hecho que supone cambios radicales en espacios y tiempos, y supone también un nuevo rol: la crianza, que compromete a todas las demás adquisiciones (emancipación económica y domiciliar plena, por ejemplo). Ciertamente, pues, la natalidad supone un antes y un después en la forma de establecimiento de la vida en pareja y de los usos del tiempo. Por esto ha sido vista como la antesala del paso a responsabilidades de adulto propiamente dichas. A nuestro modo de ver, no obstante, la centralidad continúa encontrándose en la emancipación económica (trabajo) y familiar (nuevo domicilio).

31. Conviene entender por adscripción el resultado de la socialización en el sentido de aprendizajes e interiorizaciones; por enclasamiento, el resultado de la estratificación social, en el sentido de posición social adquirida y potencial de movilidad, y por emancipación familiar plena, la disposición sobre un domicilio propio. Adquisición, enclasamiento y emancipación son los ejes de desarrollo de los jóvenes. P. Bordieu ha hecho una aportación muy firme en este sentido (por ejemplo: en Questions de sociologie, París, 1984), tanto en el fenómeno de las trayectorias sociales y el enclasamiento, como en el mismo concepto de juventud, que, de forma provocativa, define como "palabra", lo cual ha llevado a muchos a considerar que era muy nominalista en este aspecto (por ejemplo: B. Charlot en «Juventud y educación: Aproximaciones filosóficas y sociológicas", en A. Marrero (comp.), Todas las escuelas, la escuela, Valencia, 2007). O. Dávila (y otros) se apoya precisamente en el concepto de trayectoria social de Bordieu para desarrollar su aportación teórica acerca de los itinerarios de los jóvenes (en Los desheredados, Chile, CIDPA, 2005). 
debían atender la contradicción entre una demanda creciente de escolarización postobligatoria y la crisis del mercado de trabajo, sobre todo en una coyuntura demográficamente poco favorable. La extensión del tiempo de permanencia en el hogar de origen tomó entonces una segunda centralidad. Es decir, los estudios y los informes, finalmente, se centraban básicamente en considerar el paro juvenil y el retraso en la emancipación familiar. Para el caso español, el informe Juventud 84, dirigido por J.L. Zárraga, resultó emblemático: se consideraba la presión demográfica de las cohortes de jóvenes sobre la oferta escolar y laboral, se consideraba que el sistema educativo postobligatorio generaba altas expectativas de movilidad social, se dictaminaba el carácter estructural del desempleo juvenil y se anunciaba la prolongación de los jóvenes en el hogar de origen y su emancipación tardía. Todo ello (junto con la necesidad por parte del Estado de arbitrar políticas específicas) generó lo que ha venido a llamarse «estudios sobre transición a la vida activa», incidiendo sobre todo en la complejidad del tránsito de la escuela al trabajo y la necesidad de arbitrar políticas sociales de protección al desempleo juvenil. G. Welbers y B. Swartz ${ }^{32}$ han sido punta de iceberg de este tipo de informes y, sin duda, han ejercido influencia en los estudios sobre inserción profesional del GRET. No obstante, pronto hubo dificultades de orden conceptual que se aventuraban problemáticas; por ejemplo: el uso de términos como vida activa y vida adulta (muy economicista el primero y muy psicologista el segundo), o bien la acotación conceptual del ser joven y de oclusión juvenil (muy encorsetados por el término cohorte de edad y dependiente de la acción de los estados nacionales sobre los jóvenes). Otro orden de dificultades provenían de ciertas posiciones políticas que veían en el término transición ciertos apegos funcionalistas (próximos al enfoque más clásico del conflicto de roles y la asunción de responsabilidades de adulto) o ciertos apegos negativos (considerar la juventud como una etapa vacía de contenidos propios ${ }^{33}$ ).

Es por ello que una de las contribuciones del GRET ha sido ahondar en estos conceptos para formular propuestas acerca del enfoque de la transición de los jóvenes. Se propone una distinción mental entre «condición social» y «situación social», a fin de clarificar el mismo concepto de juventud. Generalmente, ha habido acuerdo común en las ciencias sociales acerca de la crítica del concepto de juventud basado en apreciaciones acerca del grupo de edades

32. G. Welbers, como gestor de los programas de transición (posteriormente programas Petra) desde la UE, y B. Swartz, como impulsor de las políticas sociales sobre inserción laboral de los jóvenes para Francia. El mencionado informe Juventud 84, de Zárraga, cumplió el mismo objetivo para España, al dar paso a una reflexión distinta acerca de los jóvenes. Los escritos de Jordi Planas i J. Casal en el marco del GRET tuvieron influjo también en este campo, tanto en Cataluña como en el resto de España.

33. El debate entre juventud como transición y moratoria positiva arranca precisamente de esta consideración. En los estudios sobre transición, se incide mucho sobre los logros (enclasamiento y posición social), y en el enfoque de la moratoria como tiempo positivo, se incide más sobre aspectos de cultura juvenil propiamente dicha (más como sala de estar que como sala de espera, como se ha dicho de forma más casera). En este sentido, véase O. Galland o bien AAVV, La nueva condición juvenil y las políticas de juventud (Barcelona, Diputació de Barcelona, 1999, Materials de Joventut). 
(dimensión etaria), distinguiendo claramente lo que proporciona un concepto social del imperativo administrativo y de los constreñimientos en el trabajo de campo ${ }^{34}$, pero la confusión conceptual se acrecienta cuando se mezclan aspectos conceptuales (o esencialistas) con aspectos derivados del cambio (sea coyuntural o del mismo capitalismo informacional), es decir, cuando hay una mezcla de lo que se entiende por juventud con los cambios en la juventud derivados del cambio social. Finalmente, parece que los «juvenólogos» se condenan a sí mismos a tomar alternativas por una conceptualización essencialista (la naturaleza de la juventud en versión positiva o negativa, integracionalista o conflictual, etc.) o por una conceptualización nominalista (juventud como puro nombre) en aras a la centralidad de los procesos sociales de enclasamiento y una relegación de la «juvenologia» a la psicología de la adolescencia. Desde nuestro enfoque de investigación sobre transición, proponemos la distinción entre condición social y situación social. La condición juvenil (naturaleza o esencia del proceso juvenil) basada en la transición profesional (escuela, trabajo y profesión) y en la transición familiar (emancipación familiar del domicilio parental al domicilio propio) supone que lo juvenil consiste precisamente en el camino (itinerario) que el joven sigue en pos de la posición social (transición profesional) y la autonomía plena (domicilio o lugar de residencia) ${ }^{35}$. La situación social de los jóvenes (cambios sociales y afectaciones sobre los procesos de transición) es cambiante y, según como, emergente; por ejemplo: determinadas modalidades de transición en el marco del capitalismo informacional pueden constituirse como dominantes (o hegemónicas) o como emergentes (significativas) $^{36}$. Es decir, por un lado, una definición sociológica de juventud como tal (doble transición, profesional y familiar) y, por otro, afectaciones del cambio social en las mismas modalidades de transición (emancipación tardía, complejidad en la transición profesional, la aproximación sucesiva como forma dominante de encarar el futuro, emergencia de la precariedad laboral, o las mismas regresiones laborales o famliares fruto de las estructuras del mercado de trabajo y de los cambios estructurales en la familia).

Sociológicamente, la juventud consiste en la realización de dos transiciones: la transición profesional y la transición familiar. La particularidad del estudio

34. La bibliografía casi resulta repetitiva. Cada investigador se ha visto obligado a precisar la noción de juventud. Lo hemos visto en el mismo P. Bordieu (o.c.). En el caso de «juvenólogos» del ámbito catalán, ocurre lo mismo. Ver, entre otros, Raül Tormos, La influencia de l'edat en el comportament electoral a Catalunya (Barcelona, Generalitat de Catalunya, 2005).

35. No pocas veces la emancipación familiar supone lugares intermedios entre la dependencia y la plena autonomía. En muchas ocasiones, hay regresiones al domicilio parental, en parte por la movilidad de los estudiantes, en parte por rupturas familiares o emocionales propias o de los padres. Esta disparidad queda reflejada en la encuesta a los jóvenes (o.c.).

36. De los dos artículos publicados en el número 79 de Papers: Revista de Sociologia, el primero («Aportaciones teóricas y metodológicas a la sociología de la juventud desde la perspectiva de la transición») incide más en los aspectos esencialistas (itinerarios de transición) y el segundo ("Changes in forms of transition in contexts of informational capitalism») incide más en los cambios en las modalidades de transición profesional y emancipación de los jóvenes. 
sociológico de la juventud precisamente estriba en el «enclasamiento y en el posicionamiento social», así como en la disparidad de segmentaciones sociales en las cuales tienen lugar las dos transiciones. Intentamos situar la construcción conceptual del término lejos del psicologismo (transición a la vida adulta) y lejos del economicismo (transición a la vida activa); orientamos el concepto hacia la "adquisición de posición social» y de «emancipación familiar». Desde esta perspectiva, la transición viene definida como el conjunto de procesos biográficos de socialización que, de forma articulada entre sí, intervienen en la vida de las personas desde que asumen la pubertad y que proyectan al sujeto joven hacia la consecución de la emancipación profesional y familiar y a la adquisición de posiciones sociales ${ }^{37}$. La transición, por tanto, se conceptúa como proceso de adquisición respecto a las relaciones de producción y reproducción (logros en estabilidad o en inestabilidad, es decir, cristalizaciones en lo profesional y en la emancipación familiar). Desde esta perspectiva, la transición tiene poco o nada que ver con los «tiempos de espera, los tiempos muertos y los tiempos finales», más bien tiene que ver con la adquisición y la resolución que atañen a las personas jóvenes. Lo que caracteriza el «ser joven» (es decir, la condición juvenil) es el proceso de adquisición (profesional y familiar), que tiene mucho que ver con el estatus social. Lo que califica la situación de los jóvenes (es decir, la situación de los jóvenes) es la complejidad de la transición en el contexto del capitalismo informacional. Consideramos dos tiempos clave.

\subsection{La transición de la escuela al trabajo}

En el planteamiento general de la transición de la escuela al trabajo, ha habido una determinación fuerte de lo político sobre lo social basado en la inmediatez del momento: cuando ha habido momentos de pleno empleo, la transición de la escuela al trabajo se percibe como inmediata (la inserción profesional de los universitarios, el empleo de los inmigrantes, la génesis de sectores económicos emergentes, nuevas empresas y ocupaciones, etc.); cuando ha habido momentos de paro juvenil, la transición se percibe como prolongada y socialmente dura. Cuando esto ocurre desde las esferas institucionales, se emite un discurso acerca de la complejidad de la transición de la escuela al trabajo.

La posición del GRET está muy lejana de este planteamiento: la transición de la escuela al trabajo es compleja por propia definición, ya que los últimos años del itinerario escolar y formativo son complejos en sí mismos (por la emergencia del trabajo de acompañamiento ${ }^{38}$, por la relación entre

37. En cuanto a terminología, L. Garrido y M. Requena aportan el término adquisición y P. Bordieu y J.C. Passeron aportan el de enclasamiento. Ambos términos los consideramos indispensables para una sociología de la juventud, sobre todo por su vinculación a la socialización y el origen social.

38. En el GRET, hemos acuñado el término trabajo de acompañamiento para identificar situaciones de empleo (irregular o regular, legal o alegal, continuado o circunstancial) por parte de jóvenes estudiantes que aspiran a empleos relacionados con sus estudios y con la calificación adquirida en el aparato escolar. 
educación y ocupación, por las estrategias de empleo, por el capital cultural y social acumulado, por las oportunidades de los mercados locales, etcétera). Y también es compleja por el tiempo de posicionamiento en la estructura ocupacional, sobre todo en momentos de alta movilidad y rotación. Es decir, definimos la transición de la escuela al trabajo no como el tiempo de paro o el vacío entre ser estudiante y ser trabajador, sino desde los dos o tres últimos años de permanencia en la escuela hasta los dos o tres años de continuidad en una posición laboral definida. Es decir, entendemos la transición como el conjunto de adquisiciones, expectativas y acciones del actor estudiante y su concreción en un proceso de posicionamiento social o enclasamiento en la estructura ocupacional (la continuidad en la posición o la calificación profesional).

Ahí, entonces, radica la centralidad de los itinerarios escolares y formativos, de los itinerarios laborales y de las probabilidades de movilidad intergeneracional neta ascendente o descendente: en la transición profesional, el sujeto joven pasa por la institución escolar (obligatoria o postobligatoria) en búsqueda de una acreditación laboral que le permita acceder de alguna forma al mercado de las cualificaciones laborales en el mercado de trabajo. Es muy posible que esta relación entre escuela y trabajo sea poco transparente, con lo cual la transición resulta cada vez más opaca. Es más, el capitalismo informacional establece más segmentaciones si cabe en la relación entre escuela y trabajo, y prolonga el tiempo de construcción en los itinerarios formativos prolongados y en la adquisición de calificación laboral.

En la transición de la escuela al trabajo, la escuela, las oportunidades y las disparidades en los itinerarios formativos tienen centralidad: son buenos indicadores la titulación conseguida, el tiempo escolar invertido, las disparidades y las interrupciones, así como el valor añadido del capital cultural. Lo mismo ocurre en el inicio de los itinerarios laborales; primeramente, con o sin trabajo de acompañamiento; posteriormente, con la toma de opciones y aprovechamiento de oportunidades de empleo, la generación y el ajuste de expectativas construidas en el entorno personal, el grupo de iguales y el entorno familiar (en definitiva, el horizonte profesional y de clase). Es claro que algunos itinerarios laborales aventuran trayectorias de futuro poco halagüeñas, lo cual define un campo para el desarrollo de políticas de inserción contra la exclusión del mercado de trabajo.

\subsection{La transición familiar}

Igual que en el caso anterior, en el planteamiento general de la transición familiar plena, ha habido una determinación fuerte de lo político sobre lo social basado en la inmediatez del momento: cuando las condiciones de emancipación se hacen más difíciles de alcanzar (coste de la vivienda, costes de oportunidad o inestabilidad en las relaciones) y la emancipación tiende a prolongarse, las instituciones tienden a presentar la emancipación como problema social. Es 
lo que ocurre a partir de los años ochenta ${ }^{39}$. Se entiende así como problema social el hecho de que el coste de la vivienda haya sobrepasado el precio umbral, o que los jóvenes adultos permanezcan en casa de sus padres sin aportar costes de mantenimiento. También se percibe como problema el desajuste de tiempos de compromisos familiares y tiempos de compromisos matrimoniales.

La posición del GRET, no obstante, consiste en vincular el hecho de la emancipación (tanto si es tardía como precoz) en clave conceptual (es decir, como neolocal). Igual que en el caso anterior, la «transición de la familia de origen a la familia propia» comprende aproximadamente los dos o tres últimos años de permanencia en la familia de origen hasta los dos o tres años de continuidad en una posición domiciliaria definida, es decir, el proceso de emancipación que supone cristalizar una forma de vida basada en el dominio sobre la vivienda y una forma continuada de relación familiar dentro de la variabilidad de formas actuales: antes, preferentemente, el matrimonio ritualizado y, actualmente, en multitud de formas de establecimiento, tanto formales como no formales.

La emancipación familiar plena es, pues, el segundo componente de la transición y supone, en nuestro contexto neolocal, el establecimiento de un nuevo domicilio u hogar propio (de alquiler o compra). Hoy día, el hecho de retrasarse en la emancipación domiciliaria merece la atención de los jóvenes por la caída de opciones de emancipación (incertidumbre económica y emocional) y por parte de sus padres por la prolongación del tiempo de permanencia en el hogar parental (muchas veces actitudes de baja colaboración o disentimiento). De ahí que las circunstancias que favorecen o retardan las expectativas y las previsiones de emancipación familiar tienen hoy día más importancia si cabe.

Si hay disparidad social en los itinerarios, también hay disparidad en las formas de emancipación: en el impulso inicial, en el tiempo de emancipación y en las modalidades de emancipación. Es decir, la emancipación familiar plena es una construcción social; puede que no sólo sea un problema del individuo y su contexto familiar ${ }^{40}$.

La emancipación familiar tiene que ver con los impulsos iniciales o las motivaciones que llevan al joven a tomar medidas económicas y personales complejas para asumir el hecho de la emancipación real. El impulso puede ser variable: la emigración, la ruptura familiar o el conflicto parental, la constitución de vida en pareja o la edad relativa en la que la promoción asume mayoritariamente la emancipación. En segundo lugar, la emancipación familiar plena se asume por modalidades distintas: o por nupcialidad anunciada, o por cohabitación o en forma grupal o individualmente. Cuando existe motivación o impulso inicial, los jóvenes empiezan el proceso de emancipación que se

39. Sobre este aspecto, conviene distinguir entre el problema social, el problema político y el problema sociológico, tal como hemos escrito recientemente (Joventut i polítiques de joventut, 25 aportacions, Diputació de Barcelona, 2007).

40. AAVV, Joventut i politiques de joventut: 25 aportacions, Barcelona, Diputació de Barcelona, 2007. 
consolida con el acceso a vivienda propia y, finalmente, con variaciones importantes en el espacio y en los tiempos de la vida cotidiana (básicamente, con la asunción de roles de crianza en la natalidad ${ }^{41}$. De nuevo, entonces, conviene distinguir entre los aspectos de naturaleza (el hecho mismo de la emancipación familiar en contextos neolocales) de los cambios sociales que afectan al proceso actual de emancipación (variabilidad en la forma y en el retraso en la edad de emancipación plena). De ahí, entonces, que convenga entender la emancipación familiar como un proceso de los jóvenes en un contexto dado y que el retraso o adelanto de la emancipación se debe a la concurrencia de causas (precio umbral de la vivienda, horizonte de la emancipación o ritualización).

\section{Transiciones, itinerarios y trayectorias}

Las dos transiciones de los jóvenes (transición profesional y emancipación familiar plena) están constreñidas por el contexto social, por las elecciones del sujeto, por las probabilidades de resolución, por la acumulación de experiencias vitales significativas, por la proximidad de políticas sociales de transición y, obviamente, por el origen social familiar, de ahí el trabajo del sociólogo en expresar las desigualdades sociales en las desigualdades de logro (contricciones sociales y elección racional). Es por esto que la reconstrucción de los itinerarios de los jóvenes ${ }^{42}$ (la disparidad social de los itinerarios) ha resultado clave para el estudio de las modalidades de transición y, para ello, la metodología de corte longitudinal se ha revelado muy fecunda.

El objeto de la sociología de la juventud, finalmente, no consiste en describir las situaciones sociales de los jóvenes afectados por las dinámicas del capitalismo, sino en identificar los itinerarios básicos que describen los jóvenes en la toma de decisiones y oportunidades acerca de la transición profesional y la emancipación familiar, así como en establecer las relaciones con la estructura social y la construcción de expectativas y oportunidades. Identificar los itinerarios y las probabilidades de transición en éxito o fracaso social es el objeto de la sociología de la juventud. De ahí entonces la centralidad de los términos itinerario y trayectoria: itinerario como expresión de un recorrido realizado y trayectoria como futurible anunciado (el momento actual como probabilidad por parte de la sociología y la intervención como posibilidad por parte

41. En otros lugares, nos hemos referido al impacto de la natalidad en términos de espacio y tiempo y en términos de roles en torno a la crianza. Es obvio que la natalidad supone transformaciones en las responsabilidades que afectan a expectativas y actitudes. Teniendo en cuenta que hay un cierto retraso en la natalidad, también hay cierta prolongación de actitudes y expectativas atribuidas generalmente a los jóvenes (básicamente, desafección de espacios y tiempos respecto a la familia de origen). Las acciones de los jóvenes varían ante la crianza; en su defecto, varían ante el grupo de iguales (muchas veces, jóvenes sin emancipación familiar, con soltería definitiva o natalidad retardada, incorporan lentamente acciones propias de los pares, tanto en espacios como en tiempos).

42. Una mayor explanación acerca de los itinerarios de transición puede leerse en los dos artículos de los autores en Papers: Revista de Sociologia, número 79 (monográfico sobre materiales de juventud). 
de las políticas de transición). La vía utilizada para conseguir informaciones plausibles acerca de los recorridos biográficos ha sido el análisis longitudinal retrospectivo ${ }^{43}$ como metodología que se ha revelado adecuada y fecunda, ya que permite reconstruir los itinerarios escolares y formativos desde la pubertad, registrar los primeros escarceos laborales hasta consolidar una ocupación (o la falta continuada de ocupación), identificar la posición inicial (muy diferente a la situación de primer empleo tantas veces utilizada como indicador suficiente por parte de la evaluación de políticas de empleo) y tener referentes acerca de los modalidades y las expectativas de emancipación familiar plena. El enfoque longitudinal tiene mucho sentido en el estudio sociológico de la juventud.

Es por ello que la propuesta GRET acerca de la transición articula aspectos de esencia (o naturaleza social del ser joven) con el estudio de los cambios derivados del nuevo capitalismo informacional sobre la juventud a partir del itinerario recorrido (familiar, escolar y laboral) y de la trayectoria (o itinerario probable).

Del estudio de los itinerarios transcurridos en jóvenes adolescentes y adultos (siguiendo la terminología de Allerberck y Rosenmayr ${ }^{44}$ ), hemos tenido oportunidad de identificar la disparidad social en los itinerarios escolares: los que pronto rehuyen la propuesta escolar, los que la rechazan, los que se sumergen en trayectos de largo recorrido y los que circulan sólo en las enseñanzas postobligatorias no universitarias. Ocurre igualmente en la inserción laboral, marcada por itinerarios de movilidad ascendente o descendente a partir de una ralentización del valor de cambio de los títulos, del trabajo de acompañamiento de los estudiantes mayores de edad y de los escarceos ante el mercado de trabajo de los jóvenes universitarios, en definitiva, de disparidad en los logros profesionales (de los logros y de las expectativas creadas ante ellos y de las acciones individuales o colectivas hacia ellos y del capital social acumulado o de origen).

Asimismo, la consideración de la teoría de la segmentación del mercado de trabajo ha resultado ser de un gran valor, ya que la base de la sociología de la juventud, a nuestro modo de ver, no es otra cosa que una sociología de la posición social y del enclasamiento. De ahí que la relación entre educación y trabajo haya sido el eje iluminador de nuestro peregrinaje en busca de itinerarios y trayectorias a comprender.

Así, con el bagaje del enfoque de la transición profesional y familiar basado en los itinerarios y las trayectorias, la aportación de lo longitudinal y de estudios sobre segmentación social, es posible construir una sociología de la juventud para una sociedad en cambio informacional. Este es el objeto del GRET en esta línea de investigación acerca de las transiciones de los jóvenes:

43. La encuesta longitudinal dice bastante acerca del historial juvenil anterior, pero poco acerca de efectos de futuro sobre trabajo y emancipación. De ahí que el sistema "panel» sea muy adecuado para el estudio de las transiciones, aunque se trate de una técnica muy gravosa económicamente y técnicamente.

44. Allerberck y Rosenmayr, o.c. 
conocer los mecanismos de construcción actual de la profesionalidad en el paso de la escuela al trabajo y la construcción actual de la emancipación familiar plena.

Parece que el enfoque de la transición tienda a lo cualitativo, a diferencia del empiricismo propio de las encuestas sobre la juventud ${ }^{45}$; por lo biográfico, por el carácter subjetivo de la comprensión de los itinerarios, por la consideración de un itinerario recorrido complejo, o, simplemente, por los costes del trabajo de campo cuantitativo ${ }^{46}$. Hace mucho tiempo, el mismo Boudon escribió acerca de la falacia epistemológica de contraponer métodos (la falsa querella del mètodo ${ }^{47}$ : el carácter longitudinal es lo que determina el enfoque de la transición, tanto si la opción és cuantitativa como cualitativa. Lo longitudinal ha caracterizado el uso de técnicas de recogida de datos, sea en base a encuestas por muestreo, sea por entrevistas en profundidad, sea por medio de grupos de discusión. En los próximos meses, la línea de investigación señalada va a acometer un análisis socioestadístico de la extensa encuesta realizada por el INE a la promoción que dio por terminados los estudios en el año 2001 y que lleva bastante tiempo en el mercado laboral o en las relaciones de trabajo: la encuesta ${ }^{48}$. Con anterioridad, otras encuestas por muestreo han permitido realizar análisis retrospectivos de los itinerarios, pero el uso de técnicas cualitativas (grupos de discusión y entrevistas en profundidad) ha sido continuado ${ }^{49}$.

\section{Resumen y perspectivas}

El enfoque de la transición (profesional y familiar) junto con la metodología longitudinal promete desarrollos de la sociología de la juventud en los próximos años. Terminamos este escrito con una referencia a ello en término de perspectivas de futuro e implicaciones en el campo de la investigación social (obviamente, hay implicaciones en orden a las políticas sobre juventud, que aquí omitimos aunque sea en desagrado). Nos referimos, entonces, sólo a algu-

45. J. Estruch y S. Cardús escribieron hace mucho tiempo un alegato a la falta de teoría y análisis socioestadístico de gran parte de las encuestas a los jóvenes en Cataluña en el primer quinquenio de los años ochenta: Les enquestes a la joventut, Barcelona, Generalitat de Catalunya, 1984.

46. Muchos sociólogos en formación (tesis doctorales en curso) que optan por estudiar a la juventud siguiendo las pautas teóricas de la transición y de los itinerarios optan por registros cualitativos (grupos de discusión, entrevistas semiestructuradas, discursos y biografías) ante las dificultades de los métodos cuantitativos para captar tales indicadores, por los costes del trabajo de campo u otros impedimentos.

47. R. Boudon, Los métodos en sociología, Barcelona, Akal, 1973.

48. La línea de investigación sobre transición explicitada acomete actualmente una explotación de la encuesta ETEFIL del INE, realizada en el año 2005, acerca de la inserción laboral de jóvenes que han terminado o que han abandonado los estudios con cinco años de anterioridad. Esta investigación se realiza mediante fondos I+D+I del MEC.

49. La inserción social y profesional de los jóvenes; La inserción de hombres y mujeres de 31 años de edad; Enquesta als joves de Catalunya 2002; L'absentisme; La formació professional; La transición 16-19 en España. 
nas implicaciones en el campo de la investigación social; seleccionamos cinco que son objeto constante de alimentación en la línea de investigación que hemos tratado de presentar.

a) Aquisición y enclasamiento son dos términos muy apropiados a la sociología de la juventud. El enfoque biográfico proporciona, además, aspectos de proceso de socialización en lo individual y lo colectivo. La perspectiva de los itinerarios escolares, profesionales y familiares es fecunda y prometedora.

b) Las teorías de la segmentación del trabajo han supuesto un marco teórico para interpretar la disparidad de los itinerarios laborales de los jóvenes. Ciertamente, se impone una redefinición de segmentos y procesos, pero el enfoque de fondo permanece.

c) La perspectiva de los itinerarios aporta la elección del sujeto en clara constricción de opciones y logros; el enfoque generacionalista contribuye al análisis del cambio en los procesos de socialización y familia; el enfoque del ciclo vital destaca la especificidad de lo juvenil respecto a otros estadios del desarrollo personal. La perspectiva de los itinerarios no sólo permite mayor ajuste a comprender la realidad actual, sino que complementa limitaciones de los otros enfoques.

d) La metodología longitudinal es básica para el enfoque de los itinerarios, pero resulta de interés para el estudio de las generaciones y de los ciclos vitales. No obstante, la recogida de datos para el análisis longitudinal resulta muy compleja y muchas veces distorsiona la misma realidad. Muchas veces, los instrumentos son complejos para el análisis de itinerarios muy simples, y no pocas veces los datos acerca de la inserción profesional tienen sesgos y limitaciones territoriales.

e) La metodología cualitativa no se entiende como una alternativa (más simple y con costes más aceptados), sino como complemento de limitaciones de estudios cuantitativos necesarios (o estadísticas regulares o encuestas por muestreo representativas). La prospección acerca de itinerarios sin logros sociales es un reto ante los procesos de riesgo de exclusión social.

f) Dudamos ante la encerrona que supone un enfoque simplemente subculturalista de lo juvenil como diferenciado de lo "propio" de la adultez.. Ciertamente, la prolongación de los tiempos de transición junto con la autonomía relativa lograda por las generaciones actuales y el impacto de las tecnologías de la comunicación supone impactos y cambios en las percepciones, las actitudes y los logros, pero la clave de lo juvenil reside en la misma construcción de los lugares y las posiciones sociales, es decir, los logros sociales y las posiciones adquiridas en las parrillas.

g) Más allá de los nominalismos y los esencialismos propios de la reflexión de las ciencias sociales acerca del concepto de juventud, lo que resulta clave es el enfoque sociohistórico de la juventud, plenamente vinculado al crecimiento de las sociedades y sus contextos. De ahí que diversas juventudes «nacionales» sean de difícil comparación e interpretación, porqué obedecen a contextos sociales distintos, a estructuras sociales distintas y a expectativas 
distintas. Se pueden contrastar itinerarios similares, pero no tiene sentido realizar una comparación de los mismos sin más.

b) Temas clásicos sobre juventud siguen dominando el campo de las ciencias sociales, como los valores (el cambio y la crisis de valores), la participación (la desafección política e institucional), el consumo (la submisión a las pautas de consumo de algunos productos) o las expectativas sociales y la ciudadanía. No obstante, nos parece que el estudio de la transición profesional y familiar explica en gran parte los cambios del contexto y la adecuación de los sujetos.

i) La generalización de la escolarización prolongada y de la formación a lo largo de la vida va a afectar sobremanera a la ubicación de los jóvenes en las modalidades de transición. La "aproximación sucesiva» probablemente sea simplemente el resultado de la gestión de lo incierto.

j) La polarización social va a tener su impacto en las zonas nucleares y ricas. Los procesos de inmigración a las aglomeraciones urbanas supondrá, junto con la crisis económica y la globalización, cambios importantes en las modalidades de transición.

De hecho, se trata de un catálogo (un decálogo) para la continuidad de los estudios acerca de los jóvenes, las juventudes y las transiciones, tanto de zonas nucleares y ricas, como de las zonas emergentes y de las zonas de exclusión plena.

\section{Referencias bibliográficas}

AAVV (1985). «Joventut 1985». Papers: Revista de Sociologia, 25.

AlLERBERCK, Klaus y ROSENMAYR, Leopold (1970). Introducción a la sociología de la juventud. Buenos Aires: Kapeslusz.

BAUMAN, Zygmunnt (2000). Modernidad líquida. México: FCE.

Boudon, Raymond (1973). Los métodos en sociología. Barcelona: Akal.

Bordieu, Pierre (1984). Questions de sociologie. París: Colin.

BRUllet, Cristina; CASAL, Joaquim; MaSjuAn, Josep Maria y Planas, Jordi (1989). Los hombres y las mujeres de 31 años. Barcelona: UAB.

BECK, Ulrich (comp.) (1997). Hijos de la libertad. México: FCE.

CARLOT, Bernard (2007). «Juventud y educación: Aproximaciones filosóficas y sociológicas». En: MARrero, Adriana (comp.). Todas las escuelas, la escuela. Valencia: Germania.

CASAL, Joaquim (1996). «Modos emergentes de transición a la vida adulta en el umbral del siglo XXI». Revista Española de Investigaciones Sociológicas (REIS), 75, 295316.1996

CASAL, Joaquim; MASJUAN, Josep Maria y Planas, Jordi (1991). La inserción social y profesional de los jóvenes. Madrid: CIDE.

CASAL, Joaquim; Merino, Rafael; GARCIA, Maribel y QueSADA, Miguel (2006). «Aportaciones teóricas y metodológicas a la sociología de la juventud». Papers: Revista de Sociología, 79, 21-48

- (2006). "Changes in forms of transition in contexts of informational capitalism». Papers: Revista de Sociologia, 79, 195-223. 
Casal, Joaquim; Merino, Rafael; Garcia, Maribel y Quesada, Miguel (2004). Enquesta als joves de Catalunya 2002. Barcelona: Generalitat de Catalunya.

CASAL i altres (2007). Joventut i politiques de joventut; 25 aportacions. Barcelona: Diputació de Barcelona.

DÁvila, Oscar (2005). Los desheredados. Valparaíso: CIDPA.

ERIKSON, Erik (1989). Identidad, juventud y crisis. Madrid: Taurus.

Estruc, Joan y CARdús, Salvador (1983). Les enquestes a la joventut de Catalunya. Barcelona: Generalitat de Catalunya.

FEIXA, Carles (1993). La joventut com a metáfora. Barcelona: Generalitat de Catalunya. Galland, Olivier (1991). Sociologie de la jeunesse. París: Colin.

GARRIDO, Luis y REQUENA, Miguel (1996). La emancipación de los jóvenes. Madrid: INJUVE.

GidDENS, Anthony (1989). Sociología. Madrid: Alianza.

Horton, Paul y Hunt, Chester (1986). Sociología. México: McGraw-Hill.

LÓpez-Blasco, A. (2006). «La familia como respuesta a las demandas de individualización». Papers: Revista de Sociologia, 79, 263-284.

MaCHADO, José (2007). Chollos, chapuzas y changas, Barcelona: Anthropos.

MARTí, Carles y otros (1999). Les politiques afirmatives de joventut. Barcelona: Diputació de Barcelona.

MEAD, Margaret (1970). Cultura y compromiso. Buenos Aires: Granica.

MENDEL, Gerard (1973). El manifiesto de la educación. Madrid: Siglo XXI.

Merino, Rafael y GARCIA, Maribel (2007). Itineraris de formació laboral dels joves. Barcelona: Fundació Jaume Bofill.

Noguera, José (2003). «Quién teme al individualismo metodológico?». Papers: Revista de Sociologia, 69, 101-132.

RAFFE, David (2007). The concept of transition on system. Gent. Holland

RAPPOPORT, Louis (1986). La personalidad desde los 13 a los 25 años. Buenos Aires: Paidós.

Reguillo, Rosana (comp.) (2001). Tiempos de híbridos. México DF: Instituto de la Juventud.

RosZACK, Theodore (1972). El nacimiento de una contracultura. Barcelona: Kairós.

SCHWARTZ, Bertrand (1984). La inserción social y profesional de los jóvenes. Madrid: INJUVE.

Tormos, Raül (2005). La influència de l'edat en el comportament electoral a Catalunya. Barcelona: Generalitat de Catalunya.

WALTER, Andreas y SAUBER, Barbara (2006). «De-standardsided pathways to adulhood». Papers: Revista de Sociologia, 79, 241-262.

ZARRAGA, José Luis (1984). La inserción profesional y social de los jóvenes. Madrid: Ministerio de Cultura.

ZeITLING, Irving (1970). Ideología y teoría sociológica. Buenos Aires: Amorrortu. 